\title{
EVALUATION OF BLEACHING ON A CAD/CAM HYBRID CERAMIC MATERIAL
}

\author{
BOGDAN CULIC ${ }^{a}$, CRISTINA GASPARIK ${ }^{a *}$, MIHAI VARVARA ${ }^{a}$, \\ CARINA CULIC ${ }^{a}$, CRISTIAN DRAGOS ${ }^{b}$, \\ LAURA SILAGHI-DUMITRESCU ${ }^{c}$, DIANA DUDEA ${ }^{a}$
}

\begin{abstract}
The objective of our study was to test the bleaching effect of two whitening gels, on stained hybrid ceramic material used for Computer Aided Design/ Computer Aided Manufacturing technology. 40 rectangular specimens 12/14/1mm were prepared form Vita Enamic blocks. All specimens were accelerated stained, and distributed in two groups. Group 1 was bleached using Opalescence PF 15\% (Ultradent, USA), group 2 was bleached using an experimental gel containing natural products. $\Delta \mathrm{E}^{*}$ (used to asses the color changes) was above the perceptibility threshold of 1.2 in $40 \%$; and the $\mathrm{WI}_{\mathrm{D}}$ (used to asses the bleaching process in dentistry) showed high units for both bleaching gels. Hybrid ceramics staining showed moderate results, while the bleaching effect was present, with no statistical difference between the discolorations produced by the two bleaching gels $(p<0.05)$. SEM images showed that the bleaching gels did not affect the surface structure of the material.
\end{abstract}

Keywords: CAD/CAM, hybrid ceramics, polymer, bleaching, color, SEM

\section{INTRODUCTION:}

In the last years CAD/CAM technologies (Computer Aided Design/ Computer Aided Manufacturing) become one of the most important component of dental medicine. The technique is orientated to the future, being mainly focused on high efficiency and standardization of prosthodontics

a Department of Prosthetic Dentistry and Dental Materials, Faculty of Dentistry, "Iuliu Hatieganu" University of Medicine and Pharmacy, Cluj-Napoca, Romania

${ }^{b}$ Department of Statistics, Faculty of Economics, Babes-Bolyai University, Cluj-Napoca, Romania

c Raluca Ripan Chemistry Institute, Department of Polymer Composites, Babes-Bolyai University, Cluj-Napoca, Romania

*Corresponding author: gasparik.cristina@umfcluj.ro 
treatment. The advantages of CAD/CAM technologies consist in the quality of the materials, industrially processed, reproducibility and low cost [1]. The available CAD/CAM systems are: "in office" systems, and laboratory systems, used in the dental lab. All CAD/CAM systems have three main components:

- A scanner - for image acquisition, transforming images into data;

- An Imaging software - data processing and restoration 3D design

- A milling unit - for transforming the virtual design into a finite product (veneers, inlays, onlays, crowns, bridges) [2,3]

Innovative materials were developed in order to fulfill researchers, clinicians and patient's demands. A great variety of dental ceramics and a large selection of composite resin materials can be found on the market. Among the advantages of ceramics are a high flexural strength and great color stability, while disadvantages are high antagonistic tooth wear and high incidence of failure due to its brittle nature [4,5]. These two parameters are much improved in the case of composite resins, but the wear of the material itself is higher. While ceramics are stiffer and harder than natural tooth structure, composite resins show lower values $[6,7]$.

Manufacturers have been developing new materials for CAD/CAM systems in order to combine the advantages of ceramic materials (durability, color stability) with the ones of composites (improved flexural strength and low abrasiveness) [5,8]. Recent materials like Vita Enamic (Vita, Bad Sackingen, Germany) represent such great improvements. The material has a three-dimensional interconnected geometry with two penetration networks. One network is a glass ceramic material and the other a polymer (methacrylate for dental applications), with a proportion of $84 \%$ and $16 \%$ respectively (by weight). Material indications are for long term restorations in the anterior and lateral areas for full coverage crowns, veneers, inlay and onlays $[5,7,8]$.

Nowadays, dental bleaching is one of the most current treatments in dentistry. The treatment provides higher self-esteem to patients, with minor consequences to the teeth if correctly performed. During bleaching procedure restorative materials are bleached together with the tooth structures $[9,10]$.

The effects of bleaching agents on the surface of composite restorative materials have been studied over the years. Different bleaching products and systems have been used for at home and in in-office procedures, containing up to 38 percent hydrogen peroxide. However, 10 to $15 \%$ carbamide peroxide bleaching agents are the most widely used as at home products. The literature contains several reports of their safety and effectiveness of hydrogen peroxide releasing agents like carbamide 
peroxide or sodium perborate [11]. Unfortunately the bleaching procedure can be responsible for causing alterations of enamel surface, or dental materials, and tooth sensitivity [12]. Several studies showed that nanofile and hybrid composite resins are affected by the bleaching gel $[13,14]$. Other studies observed that the effect on the surface of the restorative materials were material dependent. The effects to the pulp were also studied, but with no clinical significance $[15,16]$.

Development of the new tooth bleaching agents based on natural products with minimal side effects on tooth structures and dental materials is beneficial in order to minimize the side effects, but only if it's clinically effectiveness is demonstrated.

In our study we evaluate the chromatic changes produced by two bleaching gels with different chemical composition, on a new CAD/CAM hybrid ceramic material. No studies were published so far, to evaluate CAD/CAM hybrid ceramics bleaching effect. The objective of our study was to test the bleaching effect of two whitening gels, on stained hybrid ceramic CAD/CAM material.

\section{RESULTS AND DISCUTTION}

40 Vita Enamic (VE) specimens were accelerated stained, and distributed in two groups $(n=20)$. Group 1 was bleached using Opalescence $\mathrm{PF},(\mathrm{OP})$, group 2 was bleached using a non-peroxide experimental gel containing natural ingredients (EG). The CIEL ${ }^{*} a^{*} b^{*}$ color parameters of the VE specimens were measured at baseline, after staining and after bleaching, using Vita Easy Shade Spectrophotometer (Vita, Germany). Color variation obtained after staining $\left(\Delta \mathrm{E}_{1}\right)$ and after bleaching $\left(\Delta \mathrm{E}_{2}\right)$, were calculated. $\Delta \mathrm{E}^{*}$ mean values for the CIELab color space and the $\mathrm{WI}_{\mathrm{D}}$ (whitening index for dentistry) were also calculated in order to assess color parameters after staining and after bleaching (Table1).

Table 1. Color changes $\left(\Delta E^{*}\right)$ and Whitening Index (WID).

Mean values and standard deviation

\begin{tabular}{|c|c|c|c|c|c|}
\hline & \multicolumn{2}{|c|}{$\Delta \mathrm{E}^{*}$} & \multirow{2}{*}{$\begin{array}{c}\mathrm{WI}_{\mathrm{D}} \\
\text { after } \\
\text { staining }\end{array}$} & \multirow{2}{*}{$\begin{array}{c}\mathrm{Wl}_{\mathrm{D}} \\
\text { after } \\
\text { bleaching }\end{array}$} & \multirow[b]{2}{*}{$\mathrm{p}$-value } \\
\hline & Staining & Bleaching & & & \\
\hline $\begin{array}{l}\text { Group } 1 \\
\text { (OP) }\end{array}$ & $\begin{array}{c}1.668 \\
(0.742) \\
\end{array}$ & $\begin{array}{c}1.687 \\
(0.608)\end{array}$ & $\begin{array}{c}21.29 \\
(0.946)\end{array}$ & $\begin{array}{c}23.85 \\
(0.612)\end{array}$ & $<0.001$ \\
\hline $\begin{array}{l}\text { Group } 2 \\
\text { (EG) }\end{array}$ & $\begin{array}{c}2.195 \\
(1.403)\end{array}$ & $\begin{array}{c}1.878 \\
(0.946) \\
\end{array}$ & $\begin{array}{c}20.71 \\
(2.050)\end{array}$ & $\begin{array}{c}23.59 \\
(1.049)\end{array}$ & $<0.001$ \\
\hline
\end{tabular}


A powerful correlation (Pearson coefficient $R=0.819$ ) and a significant statistical correlation $(p<0.001)$ were obtained between the staining and the bleaching effect of all VE samples figure 1.

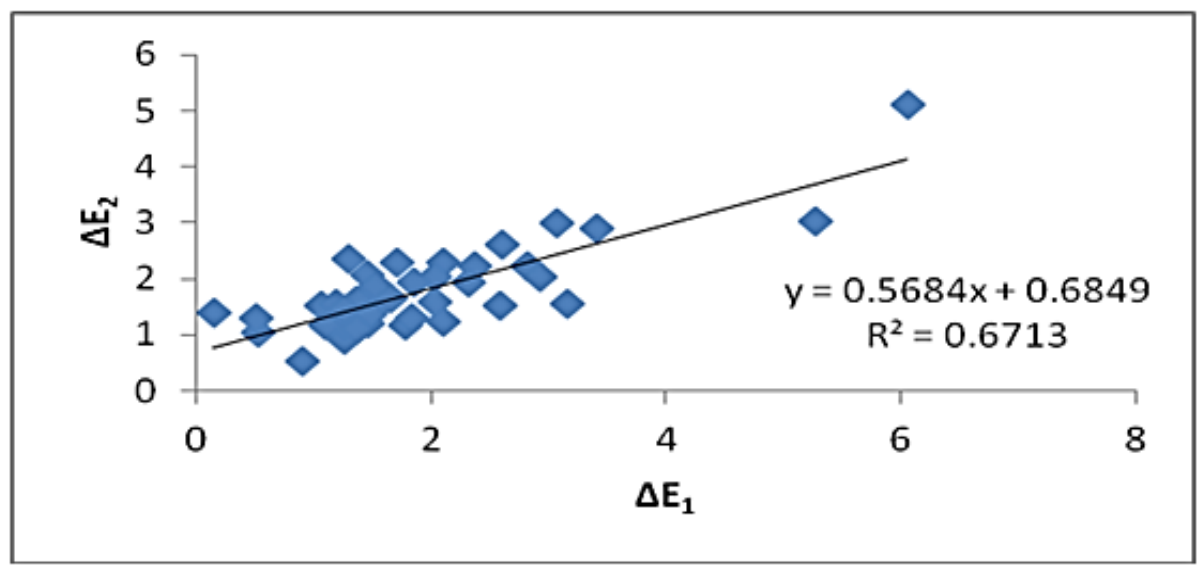

Figure 1. Correlation between $\Delta \mathrm{E}$ values obtained after staining and bleaching $\left(\Delta \mathrm{E}_{1}\right.$ and $\left.\Delta \mathrm{E}_{2}\right)$

The staining effect was visible for $17.5 \%$ of the samples $\left(\Delta \mathrm{E}^{*}\right.$ above the acceptability threshold of 2.7).

$40 \%$ of $\Delta \mathrm{E}^{*}$ (used to asses the discoloration process) were above the perceptibility value of 1.2 . However $90 \%$ were below the acceptability threshold of 2.7, fig. 2a.

The WID showed similar bleaching effect with an average of 23.85 units (st.dev. 0.612) for the OP gel, and 23.59 units (st.dev. 1.049) for EG gel. (fig. 3) The existence of a hypothesis that where is a statistical differences between the whitening effect produces by the OP and EG gels was rejected $(p=0.310)$, fig. $2 b$.

$\Delta L^{*}$ was also calculated in order to indicate the increasing or decreasing of lightness for bleaching - fig. 2c.

The SEM images at baseline, after staining and after bleaching performed at different magnifications indicates that the surface of the samples showed a complex structure, with a uniform distribution of the polymer filler and ceramic network. The red arrows indicates the organic phase, the yellow arrows the inorganic phase fig. (4-7). 
EVALUATION OF BLEACHING ON A CAD/CAM HYBRID CERAMIC MATERIAL
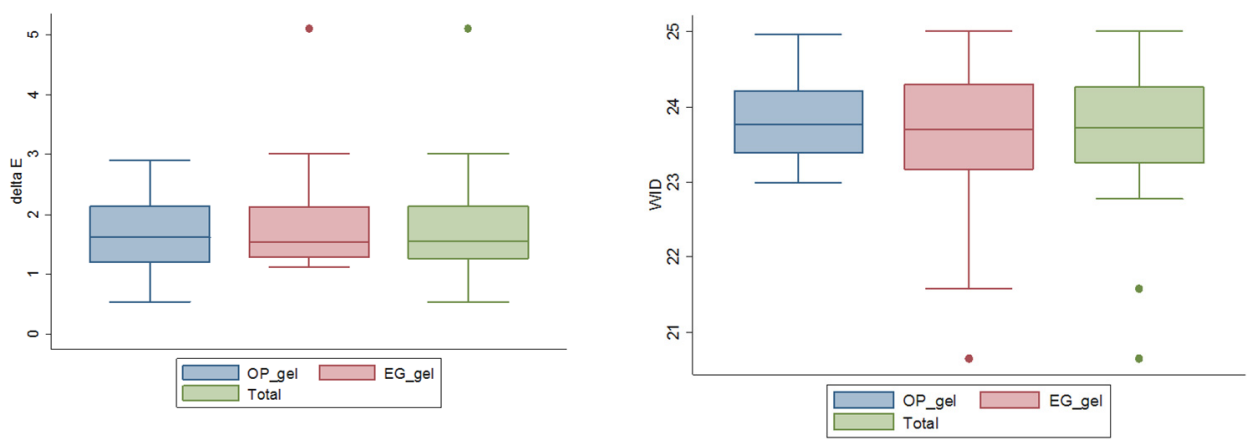

Figure 2a. $\Delta E^{*}$ values found after bleachnig for the two groups.

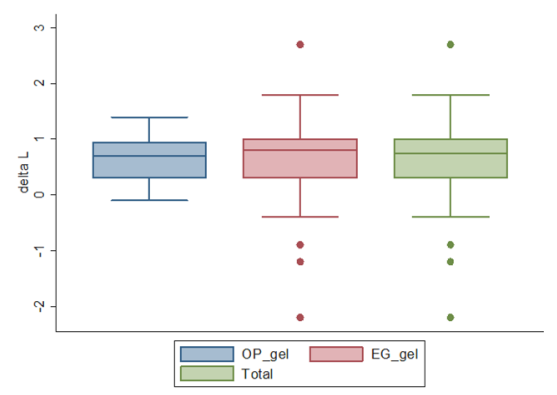

Figure 2b. Wl $I_{D}$ values for the two groups

Figure 2c. $\Delta \mathrm{L}^{*}$ after bleaching
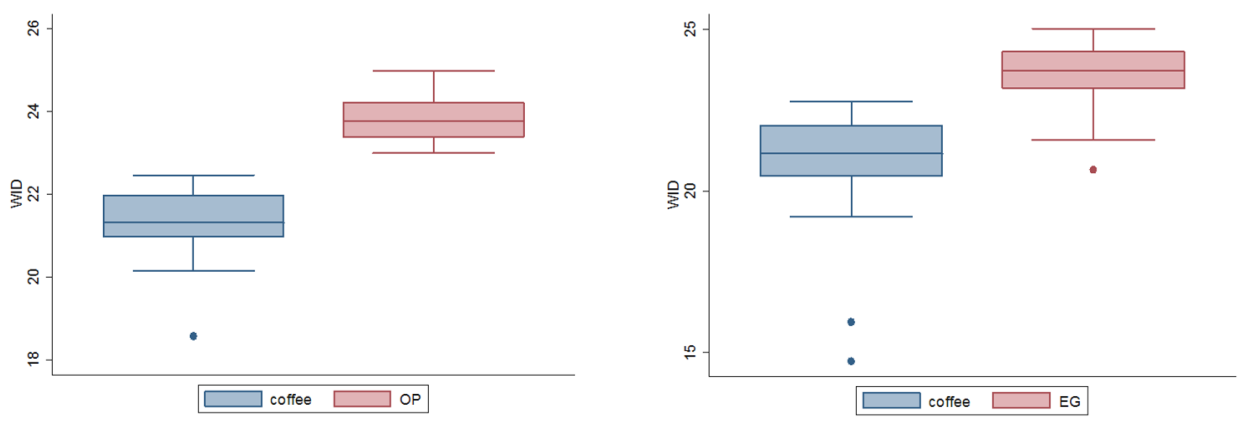

Figure 3. Wl ${ }_{D}$ after bleaching the stained hybrid ceramic 
B. CULIC, C. GASPARIK C, M. VARVARA, C. CULIC, C. DRAGOS, L. S.-DUMITRESCU, D. DUDEA
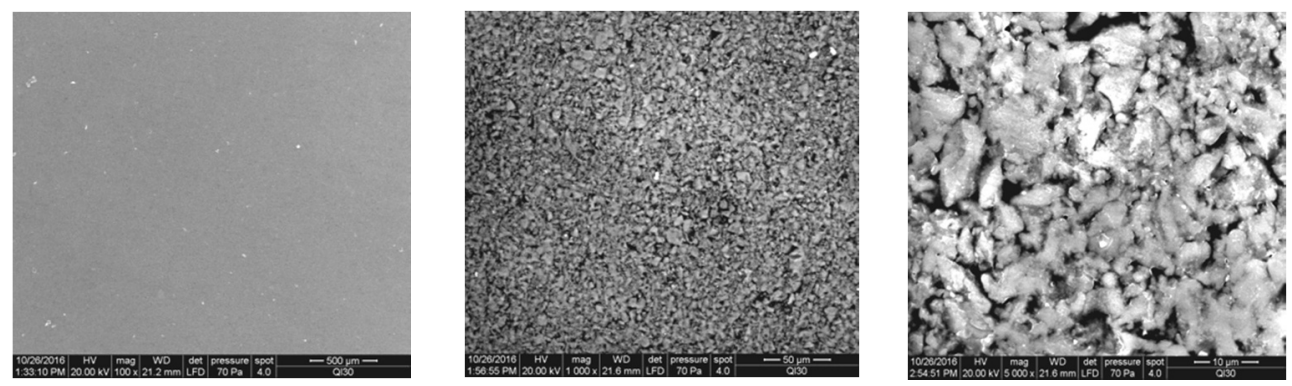

Figure 4. SEM examination at magnification of 100, 1000 and 5000 - at baseline
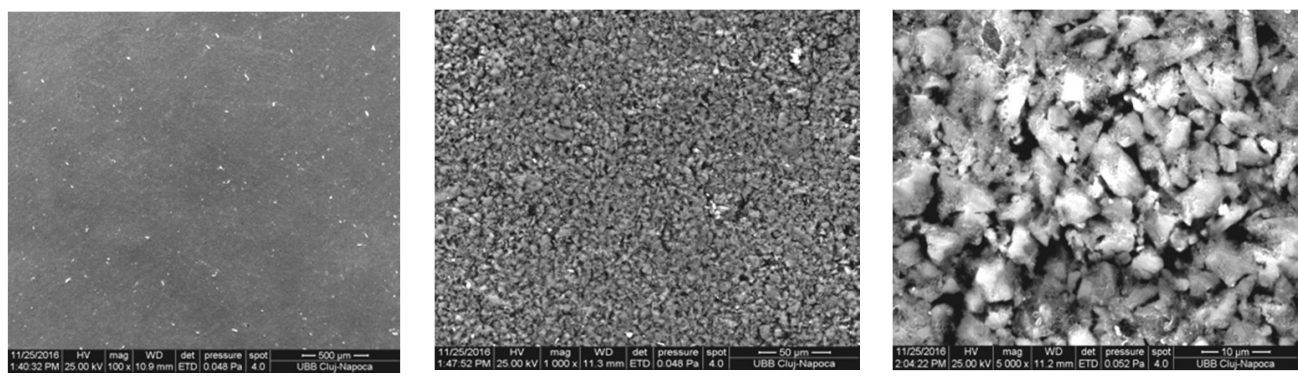

Figure 5. SEM examination at magnification of 100, 1000 and 5000 - after staining
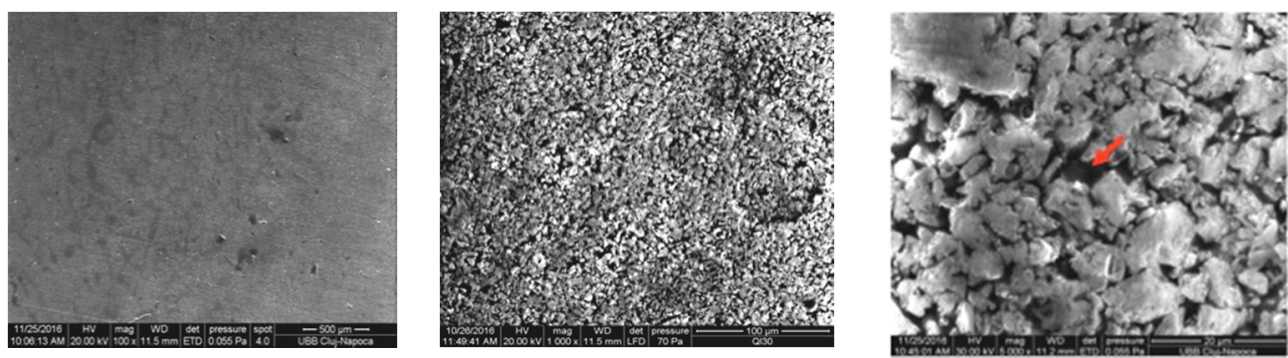

Figure 6. SEM examination at magnification 100, 1000 and 5000 - after bleaching with OP gel
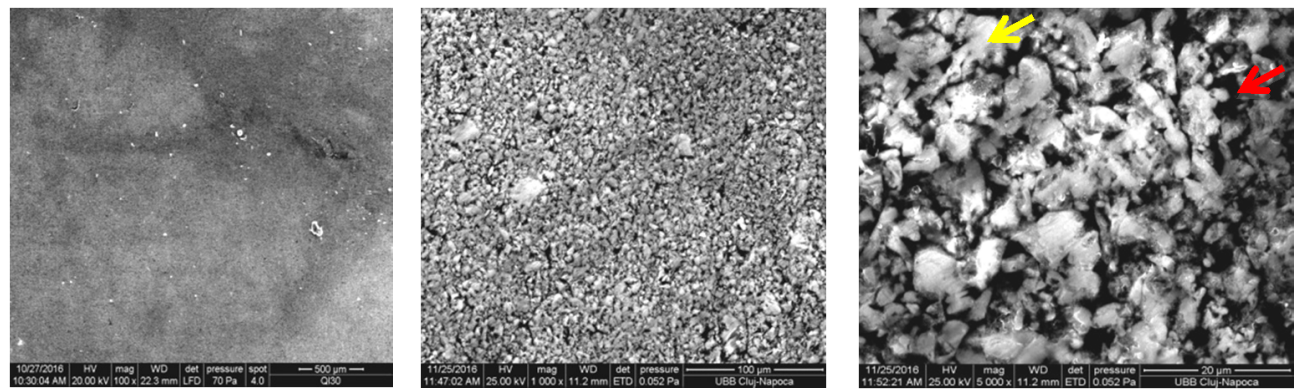

Figure 7. SEM examination at magnification 100, 1000 and 5000 - after bleaching with EG gel 
The purpose of our in vitro study was to evaluate the effect of the two bleaching gel on the surface of CAD/CAM hybrid ceramic material. Discoloration was assessed using an instrumental method (spectrophotometer), used to obtain $L^{*}, a^{*}, b^{*}$ values from the ClELab* color space, with high level of accuracy and repeatability [17].

During color measurements both light and the color of the background under which the sampled will be measured can influence the result $[20,21]$. In our study a standardized grey back was used for the measurements standardization [22].

CIELab* color space is composed by the positive $x$ axis that extends out from the center toward its maximum value of +60 at red is named the $a^{*}$ axis. Perpendicular to this and going toward the yellow hue is the positive $y$ axis called $b^{*}$. The $-a^{*}$ axis extends toward green and $-b^{*}$ toward blue. The value (or lightness) axis which is the $z$ axis extends upward with values ranging from 0 (black) to 100 (white) and is given the name $L^{*}$ [23]

CIELab* color difference $\Delta \mathrm{E}^{*}$ is a value introduced to evaluate color changes. $A \Delta E^{*}$ below 1.2 is not perceptible to human eyes; while values up to 2.7 are considered clinically acceptable. $[17,18]$

In order to use the $\Delta \mathrm{E}^{*}$ in bleaching evaluation, it has to be associate with $\Delta L^{*}$ calculation otherwise false results of the bleaching effect can be obtained. A decrease of $\Delta \mathrm{L}^{*}$, will show a $\Delta \mathrm{E}^{*}$ variation, that can be interpreted as bleaching, even the process was opposite. $\Delta \mathrm{E}^{*}$ calculation is a sum of perfect squares and will give always positive results, it will not indicate the direction of color variation, but only the amount.

WID was introduced in order to eliminate the inconveniences of other indices recommended for the assessment of bleaching. This index can have both positive and negative values. A greater value than the baseline will show a positive effect of the bleaching agent [18].

A positive WID correlated with delta $\Delta \mathrm{E}^{*}$ values give us the direction of color variation and also the quantitative level $[18,19]$.

Based on the testing conditions applied for our study design, the results showed a moderate staining of the hybrid CAD/CAM composite, $17.5 \%$ of the samples were above the acceptability threshold. The color of the stained specimens return to baseline, for both test groups, but without obtaining a discoloration above the acceptability values. Bleaching agents are effective to remove the staining for the hybrid ceramic when $15 \%$ carbamide peroxide or experimental gel was used. The whitening result produced by the two bleaching gels were similar, thus that bleaching agents based on natural products can be regarded as less aggressive alternatives to commercial gels based on carbamide peroxides [16]. 
The SEM microscope is a type of microscope used for the study of surface morphology, which use electrons for illuminating the sample and create a 3D image. Electronic microscopes have a higher resolution and magnification when compared to light microscopes.

Although conventional dental ceramics is considered the most inert of all dental restorative materials, their surfaces may present damages in contact with acidic fluoride gels or other solutions. In addition, the contact and possible diffusion of free radicals produced by bleaching agents can selectively extract the alkaline ions of ceramic network, causing damage $[11,24]$. The polymer network may also be affected by the erosion on the surface of the matrix and is dependent on resin matrix components and filler size $[14,25]$.

Dental restorations must be resistant to degradation caused by a broad range of solutions. Prolonged exposure to hydrogen peroxide affects the restoration surface and cause changes. In addition, an increase in the surface roughness may produce an increase in the accumulation of dental plaque, thus increasing the risk of secondary caries, periodontal inflammation $[25,26]$.

In general, roughness change of the restorative materials depends on the material, on the concentrations of whitening gels, but also on the exposure times, all these factors can cause greater roughness. After bleaching, color changes on the restorative materials can be different than the enamel response. Refinishing or replacement of these restorations may be necessary to enable regain of esthetics and to prevent microorganism colonization $[26,27,28]$.

In our study, the stained samples analyzed with SEM behaved well with no changes in the morphology of the surface of the hybrid ceramics, compared to the control. After bleaching with the 2 whitening gels (OP and EG) qualitative analysis of SEM images at magnification of 100x shows a slight deterioration of the surfaces, suggesting that the roughness of CAD/CAM hybrid ceramic changed after exposure to bleaching agents. Our results are in agreement with those of previous studies (Turker, Biskin, Yap, Wattanapayungkul) $[9,14]$.

In the staining and bleaching procedures we did not notice the existence of micro particles expelled or cracks on the surface of studied samples.

\section{CONCLUSIONS}

The bleaching effect of VE samples was present, but with moderate results. 
No statistical difference between the discolorations produced by the two bleaching gels $(p=0.310)$.

The Hybrid ceramics tested showed good color stability for accelerate staining.

Bleaching gels did not affect the surface structure of hybrid CAD/CAM ceramic.

\section{EXPERIMENTAL SELECTION:}

40 rectangular shaped specimens $12 / 14 / 1 \mathrm{~mm}$ were prepared from Vita Enamic CAD/CAM blocks 1M1 shade, high translucency (HT), EM14 size. The specimens were sectioned using a diamond-wafering blade mounted on a saw (Isomet Precision 1000, Buehler, USA), at $1.2 \mathrm{~mm}$ thickness. The samples were subsequently polished using sandpaper with grit size of 600,800 and $1200(\mathrm{CbC})$. The final polishing was performed using the manufacturer polishing kit (Vita Enamic Polishing Set, Vita, Bad Sackingen, Germany). At the end, a $1 \mathrm{~mm}$ thickness was achieved. All specimens with out-of- range dimensions $( \pm 60 \mu \mathrm{m})$ were discarded. The thickness was checked with an electronic digital caliper (Pittsburg, USA) for all samples. The specimens were ultrasonically cleaned in distilled water for 10 minutes and then were allowed to dry for 24 hours. All specimens were accelerated stained, and distributed in two groups $(n=20)$.

\section{Staining process}

The specimens were immersed in coffee, $50 \mathrm{ml}$ of coffee for each sample (prepared in a drip coffeemaker) using 32 grams of ground coffee / 8 cups of water). The immersion time was 3 hours per day in the colorant solution and the rest of the time in artificial saliva at $37^{\circ} \mathrm{C}$. The protocol was carried out for 21 days. Afterwards, they were rinsed in distilled water, air dried and stored in distilled water.

\section{Bleaching process}

Group 1 was bleached using Opalescence PF, containing 15\% carbamide peroxide (Ultradent, USA) (OP), group 2 was bleached using a non-peroxide experimental gel containing natural ingredients (EG). (Hydroxyapatite with Zn 15\% and strawberries/quinces juice). The bleaching agents were applied on test specimens 8 hours per day for 14 days (total 112 hours). 
The CIEL*a* $b^{*}$ color parameters of the VE specimens were measured at baseline, after staining and after bleaching, using Vita Easy Shade Advanced 4.0 Spectrophotometer (Vita, Germany). The measurements were performed against an $18 \%$ gray card background. Several pairs of color parameters were obtained for each sample ( $\mathrm{Lx}^{*}, \mathrm{ax}^{*}, \mathrm{bx}^{*}$ values from the CIELab color space) at baseline, after staining; after bleaching) which allowed to calculate the $\Delta$ Eab* values and the new whitening index for dentistry $\left(\mathrm{WI}_{\mathrm{D}}\right)$. [17]. The results were statistically analyzed using the SSPS 16.0 for Windows.

$E_{a b}{ }^{*}$ was calculated using the following formula:

$$
\Delta \mathrm{E}_{\mathrm{ab}}{ }^{*}=\sqrt{\left(\Delta L^{*}\right)^{2}+\left(\Delta a^{*}\right)^{2}+\left(\Delta b^{*}\right)^{2}},
$$

where $\Delta \mathrm{L}^{*}$ - is the difference in lightness, $\Delta \mathrm{a}^{*}$ - is the difference in the chromatic parameter $a^{*}$ on the red-green axis, $\Delta b^{*}$ - is the difference in the chromatic parameter $b^{*}$ on the yellow-blue axis from the CIEL* $a^{*} b^{*}$ color space.

The CIE* Lab color space WID was calculated using the formula:

$$
W I_{D}=0.511 L^{*}-2.324 a^{*}-1.100 b^{*}
$$

The samples were examined under SEM with FEI Inspect model $S$ microscope, in functional High and low vacuum, with an accelerating tension between 200V-30KV. The microscope is equipped with infrared CCD-IR camera and electrons detector, having a max resolution of 4096x3536 pixels. The measurements were recorded at baseline, after staining and after bleaching. Images were recorded in three different magnifications (100x, 1000x and 5000x). The images were obtained at three different locations for each sample.

\section{ACKNOWLEDGEMENTS}

The research was supported by the UMF "luliu Hatieganu" Cluj Napoca Internal Grant nr. 4994/10/2016.

\section{REFERENCES}

1. F. Beuer, J. Schweiger, D. Edelhoff, British Dental Journal, 2008, 204, 505.

2. S.B. Nathan, B. Aaronson Heidi, Compedium, 2008, 29, 494.

3. P. Feuerstein, S. Puri, Oral Health, 2009, 99, 65.

4. A. Coldea, M.V. Swain, N. Thiel, Dental Materials, 2013, 29, 419.

5. A. Awada A., D. Nathanson, Journal of Prosthetic Dentistry, 2015, 144, 587.

6. N.D. Ruse, M.J. Sadoun, Journal of Dental Research, 2014, 93, 1232.

7. A. Della Bona, P. Corazza, Yu Zhang, Dental Materials, 2014, 30, 564.

8. D. Fasbinder, G. Neiva, Journal of Esthetic and Restorative Dentistry, 2016, 28, 66. 
9. S.B. Turker, T. Biskin, Journal of Prosthetic Dentistry, 2003, 89, 466.

10. O. Polydorou, E. Hellwig, TM Auschill, Operative Dentistry, 2006, 31, 473.

11. S. Ourique, C. Arrais, A. Cassoni, C. Tsuzuki-Ota, J.A. Rodrigues, Brazilian Oral Research, 2011, 25, 453.

12. M. Goldberg, M. Grootveld, E. Lynch, Clinical Oral Investigations, 2010, 14, 1.

13. T. Attin, C. Hanning, A. Wiegand, R. Attin, Dental Materials, 2004, 20, 852.

14. P. Wttanapayungkul, A.U. Yap, Operative Dentistry, 2004, 29, 398.

15. A.F. Lima, A.P. Ribeiro, D.G. Soares, N.T. Sacono, J. Hebling, C.A. De Souza, Acta Odontologica Scadinavica, 2013, 71, 1319.

16. I. Baldea, D. Olteanu, A. Filip, M. Cenariu, D. Dudea, A. Tofan, C. Alb, M. Moldovan, Clinical Oral Investigation, 2016 [Epub ahead of print]. DOI:10.1007/s00784016-1882-4

17. R. Ghinea, MM. Pérez, L.J. Herrera, M.J. Rivas, A. Yebra, R.D. Paravina, Journal of Dentistry, 2010, 38, 57.

18. M.M. Pérez, R. Ghinea, M.J. Rivas, A. Yebra, A. Ionescu, R.D. Paravina, L.J. Herrera, Dental Materials, 2016, 32, 461.

19. A. Petran, T. Radu, B. Culic, R. Turcu, Applied Surface Science, 2016, 390, 1.

20. A.U. Guler, F. Yilmaz, T. Kulunk, E. Guler, S. Kurt, Journal of Prosthetic Dentistry, 2005, 94, 118.

21. R. Bagheri, M.F. Burrow, M. Tyas, Journal of Dentistry, 2005, 33, 389.

22. A. Alharbi, S. Ardu, T. Bortolotto, I. Krejci, Odontology, 2016, DOI:10.1007/s10266-016-0258-1.

23. C. Gaspari, A.G Grecu, B. Culic, M.E. Badea, D. Dudea, Journal of Esthetetic and Restorative Dentistry, 2015, 27, 285.

24. K.J. Anusavice, Degradability of Dental Ceramics, 1992, 6, 82.

25. S. Marto, C. Coito, A. Pequeno, Al. Cavalheiro, Revista Portuguesa de Estomatologia, Medicina Dentaria Cirurgia Maxillofacial, 2012, 53, 7.

26. A.A. Zaki, N.Z. Fahmy, Journal of Prosthodontics, 2009, 18, 223.

27. A.D. Vanderlei, S.P. Passos, S.M. Salazar-Marocho, S.M. Pereira, V.Z. Vásquez, M.A. Bottino, Acta Odontologica Latinoamericana, 2010, 23, 257.

28. A.V. Burde, S. Cuc, A. Radu, M.A. Rusu, C.S. Cosma, R.S. Câmpian, D. Leordean, Studia UBB Chemia, 2016, 61, 205. 
